\title{
EL TRABAJO Y EL DESENVOLVIMIENTO LABORAL POR AUTO CONFRONTACIÓN
}

\author{
O TRABALHO E O DESENVOLVIMENTO LABORAL PELA AUTOCONFRONTAÇÃO
}

WORK AND LABOR DEVELOPMENT BY SELF-CONFRONTATION

\author{
CUNHA, Daisy Moreira 1 \\ PINTO, Daniel Fabián Roca Flores 2 \\ MUNIZ, Maria leda Almeida 3
}

\section{RESUMEN}

Las verbalizaciones sobre el trabajo provocadas por técnicas de autoconfrontación pueden ser instrumentos útiles para la comprensión de la actividad laboral, siendo este proceso altamente educativo para aquel que trabaja, trayendo a su vez, un potencial para configurarse como método de investigación, al abrir posibilidades en términos de construcción de conocimientos desde una perspectiva comprensiva de los saberes y valores laborales. El artículo presenta parámetros de los abordajes de las clínicas del trabajo (en diálogo con los estudios sobre el ofício de la docencia.

Palabras claves: Autoconocimiento. Trabajo. Desarrollo.

\section{RESUMO}

As verbalizações sobre trabalho provocadas pelas técnicas de autoconfrontação podem ser compreendidas como instrumentos uteis para a compreensão da atividade laboral, sendo este processo, altamente educativo para aquele que trabalha, trazendo um potencial para se configurar como um método de pesquisa, abrindo as possibilidades em termos da construção do conhecimento desde uma perspectiva compreensiva dos saberes e valores laborais. O artigo apresenta parâmetros de abordagens das clínicas do trabalho em diálogo com estudos sobre o ofício da docência.

Palavras-chave: Autoconhecimento. Trabalho. Desenvolvimento.

\section{ABSTRACT}

Work expressions caused by self-confronting techniques can be useful instruments for understanding work activity. This highly educative process for the one who works has the potential to be a research method that opens possibilities in terms of the knowledge construction in a comprehensive perspective of knowledge and values that circulate work activity. The article presents parameters of clinical work approaches in exchange dialogue with studies on teaching as a profession.

Keywords: Self-knowledge. Work. Development.

\footnotetext{
${ }^{1}$ Universidade Federal de Minas Gerais - UFMG - Brasil.

2 Universidade Federal de Minas Gerais - UFMG - Brasil.

${ }^{3}$ Universidade Tecnológica Federal do Paraná - UTFPR - Brasil.
} 


\section{INTRODUCCIÓN}

Este trabajo tiene como objetivo discutir los abordajes del trabajo, en el cual, los procedimientos de autoconfrontación se muestran estructurantes para el análisis de la experiencia de aquellos que trabajan. El estudio de este método es interesante, por tener un carácter de transformar al observado en observador de su propia actividad e ir más allá de esa observación, dando un destino a ese dialogo comentado.

Al desarrollar el diálogo por la observación y la observación por el dialogo, se tiene en cuenta, en ese momento de la observación de sí mismo, es esta producirá un extrañamiento, el cual, podría provocar el crecimiento personal de aquel trabajador que es observado y que también es observador en momentos distintos. Así, a través de este método, el trabajador podrá generar las condiciones para auto evaluarse y adquirir artefactos que se transformen en instrumentos psicológicos y/o su desarrollo en las situaciones de trabajo, dando un destino a ese diálogo.

Esta perspectiva metodológica está presente en varios abordajes del trabajo cuyas bases epistemológicas nos invitan a comprenderlo desde un punto de vista "clínico", situado: instrucción al sósia (ODDONE, RE, BRIANTE, 1981); la ergonomía de la actividad (GUËRIN et al., 2001), la clínica de la actividad, (CLOT, 2001; CLOT \& FAÏTA, 2000), el interaccionismo socio-discursivo (BRONCKART, 2005) y el abordaje ergológico del trabajo (SCHWARTZ, 2006; SCHWARTZ \& DURRIVE, 2010, 2015), entre otros.

Estas perspectivas que discutimos en el ámbito de este artículo, se enfocan en el trabajo real, revitalizando el saber académico de los especialistas y de los técnicos, con foco en los saberes de la experiencia de trabajo, asociándolos con la investigación y la intervención, teniendo en vista la promoción de un nuevo conocimiento, el cual se pueda comprometer con la transformación de las situaciones de trabajo.

\section{LA INSTRUCCIÓN AL SÓSIA: PRINCIPIOS}

La convivencia y diálogo entre estos dos segmentos permitió comprender que los riesgos que se presentaban en el proceso productivo, provocaban actitudes en los trabajadores para la defensa de su salud, impulsándolos adquirir nuevos conocimientos, en la perspectiva de transformar sus condiciones de trabajo. La investigación sobre la construcción cotidiana de estos saberes desarrollados en el trabajo, posibilitó a los investigadores comprender mejor "lo que hacían, aquello que pensaban los operarios, así como quien era el operario, el sentido y los objetivos de sus luchas, centradas todas ellas sobre los problemas de seguridad e higiene" (ODDONE, RE, BRIANTE, 1981, p.21).

Los hallazgos de Oddone, Re, Briante (1981), llaman nuestra atención para el hecho de que la gran dificultad en la asociación entre la experiencia operaria y la psicología del trabajo era la resistencia en la medida de lo possible a la tentativa de encuadrar la experiencia operaria en el lenguaje y en un contexto científico que siempre la rechazo.

En ese sentido, "La experiencia, pensada en términos de aprendizaje individual y colectivo en la búsqueda por soluciones capaces de resolver problemas concretos que el trabajo colocaba todo el 
día en el interior de la fábrica, colocan a un operario que aprende, es decir, adquiere una experiencia no solamente en relación con la tarea en sí, mas también en el plano politico y sindical” (ODDONE, RE, BRIANTE, 1981, p. 17).

En ese entendimiento, el aprendizaje es producto de la vivencia individual, colectiva, configurando un patrimonio cultural de clase que hace que las condiciones de vida tal, sean como estamento de las condiciones generales de producción en las situaciones de trabajo en las cuales el trabajador vivencia. Así una experiencia material de tarea, al transmitir su saber para otros, el quien hace, puede adquirir igualmente un saber al relacionarse con los otros, en ese sentido, podemos afirmar que la formación informal es el fruto de dos experiencias; por un lado de la experiencia puramente y simplemente transmitida y por otra parte, de la experiencia nacida de las dificultades encontradas y superadas en el curso de la resolución de los problemas.

En ese segundo tipo de experiencia aparece un gran número de nuevos problemas aún sin solución y que a lo sumo no son percibidos, puesto que su objetivo difiere de aquello percibido en los problemas iniciales (máquina o tarea). El tomar consciencia de su existencia exige de los trabajadores un nivel de investigación que los localiza más allá de una dimension individual y de categorizaciones tradicionales que se tornaron inadecuadas (ODDONE, RE, BRIANTE, 1981, p. 59-60). Frente del saber de una clase que vive del trabajo en el contexto de los años 70 en Turín.

Con ello podemos decir, que la experiencia operaria es un abordaje global de los problemas colectivos de grupos de trabajadores, atravesados y estructurados por el juicio de valor de los mismos. En este sentido los investigadores se colocaron como propósito de hacer hablar al trabajador sobre esta experiencia e ir más allá de los comportamientos esperados por las normas que los encuadran. Así, con ello, se podría reconocer que los trabajadores hacen la experiencia en los recubiertos del proceso productivo, en las situaciones de trabajo en las cuales se encuentran, colocando como problema teórico metodológico el ¿cómo aproximarse y evidenciar esas experiencias?

La aproximación de los investigadores sobre la realidad concreta de los trabajadores se constituyó en un marco histórico en el sentido de un nuevo régimen de producción de saberes sobre trabajo, trayendo nuevas demandas sociales para la reflexión académica que caracterizó esa época.

Esta ampliación resultó en un nuevo modelo de análisis, producido a partir de la experiencia del trabajador, considerando el valor de su historia individual, colectiva y su capacidad de intervenir en la solución de los problemas que se colocaban en el trabajo; contraponiéndose a los saberes epistémicos validados en la ergonomía y en la psicología de la época, las cuales, utilizaban un método analítico para medir y evaluar los problemas de forma global y conociéndolo parcialmente, sin considerar al trabajador como sujeto ético y epistémico.

En la experiencia de las Comunidades Cientificas Ampliadas, Oddone, recurrió a la instrucción al sósia (ODDONE, 1981), técnica basada en el modelo milenario de simulación de comportamiento, con la cual, reproducir el proceso complejo de los comportamientos de los delegados, traduciéndolos en planos de comportamiento reales a las imágenes de la fábrica y de los hombres que son ligados a la experiencia personal y a la visión del mundo.

Esta técnica consiste en pedir a los sujetos dar instrucciones a un yo-auxiliar, un (Sósia) doble que debe responder a la cuestión: si existe otra persona perfectamente idéntica a ti ¿cómo tú le dirías 
para que se comportase en la fábrica, en relación a la tarea, sus camaradas de trabajo, a la jerarquía y a la organización sindical de manera que no percibamos que se trata de un doble (sósia)?, con ello, la captación de una imagen de comportamiento real, más no el comportamiento real y total del individuo, pero si, una representación sobre aquello que él hace de su propio comportamiento.

Al profundizar en esa instrucción, vamos encontrando que existe una distancia entre el comportamiento descrito y el comportamiento efectivo, así como también existe una parcela llenada por el control de los hechos y el testimonio del otro, interesándonos por el plano-programa que sirve de guía para la acción de cada uno. Desvelándose el esfuerzo por parte de aquel que da la instrucción, clareando así sus formas de hacer, el proceso inagotable que no permite ser finalizado y que está siempre en abierto.

El legado de Oddone, Re e Briante (1981) está en articular, en una confrontación permanente, los conocimientos científicos y la experiencia de los trabajadores, constituyéndose desde entonces como un indicador para nortear la comprensión de los problemas de salud y seguridad en los contextos productivos, con este objetivo desarrollaron la instrucción al sósia, de modo tal que pueda ser explicada la experiencia.

En este proceso su contribución fue substancialmente importante para el desarrollo de otras disciplinas que a lo largo de los tiempos vinieron a clarear el estudio del trabajo humano y también sobre los avances en los modelos de prevención de los riesgos utilizados en el ámbito del trabajo.

En los caminos abiertos por Oddone (1981), algunos investigadores se interrogan sobre la experiencia del trabajo concreto en situaciones de trabajo real, colocando el debate de normas como manantial político y epistemológico de los saberes producidos en y sobre el trabajo, en ese intento de hallar respuestas a tales interrogantes, desarrollaron entrevistas en autoconfrontación cuyo objetivo es provocar a verbalización sobre el trabajo y explicitarlo en relación con varias interfaces, con las cuales el trabajador mantiene relación (espacio, tiempo, colegas, equipos, etc.). Siendo importante subrayar, que al ir más allá de lo realizado, la autoconfrontación trae una intención de explicar, lo real de la actividad (actividad latente, reprimida, etc.: posible no realizado y los conflictos vitales).

\section{LA AUTOCONFRONTACIÓN EN LAS INVESTIGACIONES SOBRE EL TRABAJO DOCENTE}

Al reconocerse en las líneas anteriores la riqueza y potencial de transformación que ofrece la autoconfrontación como dispositivo de aproximación para el desvelamiento de la actividad, se propone también un contexto nuevo, en el cual, el protagonista del trabajo se convierte en un observador exterior de su actividad en presencia de un tercero, ampliándose así, las posibilidades de analizar lo vivido y revivido por él, colocándose al descubierto, no sólo aquello que él está viviendo, aquello que sólo llegó hacer o eventualmente puede hacer, así como aquello que él pueda experimentar sobre lo que es capaz de hacer.

En ese sentido, este dispositivo, se convierte en un medio trascendental para que él se conozca mejor, se apropie de aquello que es desvelado y reflexione sobre aquello vivido, sentido y dicho por él, siendo esta, una posibilidad para conocer al sujeto docente y los sentidos que él le atribuye a su hacer dialécticamente, pues la construcción del conocimiento es fomentada todo el 
tiempo por medio del diálogo en movimiento (MACHADO, 2012).

Al contar con la ayuda del investigador, quien mediante un proceso de intercambio entre él y el (los) protagonista (s) del trabajo, diálogos podrán ser generados y convertidos en nuevos instrumentos psicológicos de trabajo efectivo, con lo cual, estos hallazgos puedan ser llevados más allá del perímetro interlocutorio, que no necesariamente se presenta como espontáneo.

Así, con la autoconfrontación, según Muniz (2010) las coerciones se tornan recursos, al lograr que ese cuadro metodológico sea apropiado y modificado por los interlocutores, en esa dirección podemos pensar en un aprendizaje como fuente potencial de desarrollo, el cual, al ser abordado desde otras perspectivas como por ejemplo la ergología (SCHWARTZ, 2000), este dispositivo puede ser ampliado y relacionado para el análisis de los debates de normas, las renormalizaciones y los usos de sí, en que sujetos vivos en situaciones de trabajo experimentan, desvelan arbitrajes y patrimonios que son construidos y reconstruidos en ese medio donde el trabajador actúa.

Al ahondar sobre el uso la autoconfrontación como medio de análisis de la actividad del docente, Dounis et al (2012) realiza un estudio de arte al respecto, mapeando las producciones académicas del banco de datos de la biblioteca digital de la CAPES, encontrando y analizando seis tesis y 12 disertaciones de los Programas de Post graduación brasileras, las cuales, utilizan la autoconfrontación para analizar y fomentar el desarrollo profesional y apoyar acciones de formación continuada de profesores de educación infantil y superior.

Los hallazgos que estos investigadores presentan, proporcionan una convergencia del uso de este método con las bases epistemológicas de autores contemporáneos del interaccionismo socio discursivo (BRONKCART, 2005), además de aquellos relacionados con la clínica da Actividad (CLOT \& FAÏTA, 2000) y circunscritas a la ergonomía francesa (GUÉRIN, 2001), así como también con otras perspectivas, como es el caso de Alves (2009) quien la emplea desde la perspectiva ergológica.

Este mapeo permite resaltar el empleo de la autoconfrontación simple (ACS) en la mayoría de las investigaciones analizadas, esto acontece, frente a las limitantes que los sujetos participantes en las investigaciones colocan para el investigador, así como los recursos y medios necesarios para grabar en vídeo los diálogos, entre otras limitaciones de índole institucional que puedan presentarse, como suceden con los estudios realizados por Lousada (2005) y Alves (2009) quienes en ambos casos, ambas investigaciones sólo pudieron contar con el soporte de los audios y no de imágenes de vídeo, que por lo general caracteriza el uso de este método.

Por su parte, el aporte de Dounis et al (2012) se resalta por colocar la diversidad de aplicaciones de la autoconfrontación en articulación con diferentes focos de análisis tales como los centrados en la actividad docente sobre una perspectiva teórica; o como instrumento de cambios en el comportamiento del profesor y/o como herramienta de un proceso de formación continuada, teniendo como punto común en todos estos focos, producir reflexión y consecuentemente la transformación de la actividad profesional.

Ampliando el uso de este método en otros contextos latinoamericanos, la experiencia realiza por ROCA (2017) pionera en el uso de la autoconfrontación como recurso metodológico en el desvelamiento del trabajo del profesor universitario en el Perú, situado en un contexto de mercantilización de la educación superior, en el cual son establecidos sin discusión y considerados 
como naturales los mecanismos de control y las condiciones de trabajo que afectan la dimensión profesional, social y política de los profesores para el ejercicio de la docencia, terminan por colocar su actividad de trabajo como privada y reducida ser un prestador de servicios, por lo cual, sólo comprendido en su dimensión económica y productiva.

Esta experiencia que Roca (2017) presenta, tiene como prepuesto teórico metodológico a la ergología (SCHWARTZ \& DURRIVE, 2010, 2015), empleándose conjuntamente con ella, la autoconfrontación, así como otros métodos, que suman en la aproximación por conocer y comprender el trabajo del profesor universitario en el aula de clases, situando su actividad, en una universidad no estatal de carácter comunitario, originaria de un movimiento estudiantil, ubicado territorialmente en una zona periférica de Lima Norte y que propone un ideario educativo, social y político de formación crítica en sus estudiantes, atravesados por una Política pública de la educación superior peruana que vigencia un mercado educacional en que se compite por el alumno cliente.

Este marco en que esta universidad se encuentra inserta, corresponde a un contexto en el cual la universidad peruana, está permeada por valores mercantiles, influenciada por una La Ley Universitaria $\mathrm{N}^{\circ} 30220$ - 2014, que establece como órgano estatal de control de universidades a la Superintendencia Nacional de Educación (SUNEDU), quien a través de estándares e indicadores de gestión de calidad procuran ejercer presión para que sean cumplidas estas normas, en cuyo proceso, se van generando tensiones y produciendo desentendimientos entre los diversos colectivos de base con la dirección de esta universidad, al asumir sin una discusión democrática entre estas bases, un modelo de gestión empresarial que afecta su ideario originario e incorpora mecanismos de control coercitivos que afectan su proyecto educativo, la organización del trabajo y en particular el oficio del profesor.

En ese contexto, situamos al profesor universitario en esta universidad, en el aula de clases, por ser este lugar, el espacio donde confluye lo cotidiano y lo institucional, donde las normas antecedentes internas y externas prescriben su trabajo, pero también, se configura como el espacio, en que se producen los debates de normas y las renormalizaciones, en ese medio atravesado por mediaciones y arbitrajes, participaron de esta investigación, tres profesores de la Facultad de Ciencia e Ingeniería (Facultad con mayor población estudiantil y docente de esta universidad).

Estos profesores participantes voluntarios de esta investigación, presentan como características comunes, estar dedicados a la docencia superior por más de diez años, trabajando en la condición de contratados y bajo el régimen parcial de 20 horas semanales, dedicados sólo a la enseñanza en los semestres $\mathrm{V}$ al VII de la carrera de ingeniería; quienes en su práctica docente, enfrentan una diversidad de grupos de estudiantes en distintos turnos donde actúan, dedicados principalmente a la enseñanza de más de una disciplina diferente; regidos por una planificación curricular que prescribe los contenidos que tienen que ser enseñados y evaluados como principio que garantiza la buena calidad; pero sometidos al cumplimiento de procesos que acarrean trabajar fuera del horario de clases y en otros espacios, elaborando materiales y calificando exámenes y trabajos, así como de asistir obligatoriamente a reuniones de coordinación, sin tener todo esto adicionalmente trabajado reconocido económicamente como pago del salario recibido. Además de ser sometido a una evaluación de su desempeño por los estudiantes, a través de encuestas elaboradas por la coordinación 
académica, órgano, que a su vez ejerce una supervisión constante orientada al cumplimiento de las normas y mantener una performance en la enseñanza dentro del salón de clases.

En ese medio el profesor sólo es remunerado por el dictado de clases y contratado como prestador de servicios durante el semestre lectivo en el que va enseñar, por tanto sólo tendrá vinculo institucional por el tiempo en que está contratado, recontando beneficios y sus vínculos con los diferentes colectivos con los que interactúa, que como lo señala Chomsky (2014), esto lo convierte en un permanente buscador de trabajo.

Las limitaciones que la investigación enfrentó y que influenciaron en que el método de la autoconfrontación sea desenvuelto conformes las caracterisiticas propias de este método, fueron tanto la restricción de realizar grabaciones de imágenes; solicitadas por la universidad así como por los propios profesores participantes, quienes además solicitaron al investigador la preservación de sus identidades, siendo esto posible a través de la utilización de siglas que fueron colocadas para cada profesor (P1, P2, P3), siendo la producción de los diálogos de manera individual con cada profesor y no de forma colectiva.

Estas limitaciones iniciales, generó que sólo optemos en realizar la autoconfrontación simple (ACS). Para generar el diálogo e interacciones con cada profesor, en un primer momento utilizamos la entrevista semi estructurada y luego el método de la instrucción al sósia (IAS); siendo estos procedimientos claves para que el profesor verbalizará sobre su trabajo, el mismo que al ser transcrito generó un texto, que fue entregado a cada uno de ellos. Siendo este texto individual y que permitió configurar escenas sobre las cuales serían abordadas vía la ACS.

Para realizar la autoconfrontación, el investigador eligió el texto transcrito y producido por la IAS en situaciones de trabajo comunes, en la cual, los diálogos generados, proporcione una mayor exploración sobre aquello que estaba detrás de todo aquello manifestado. Así, se construyeron escenas, con las cuales se configuraron a partir de las similitudes y diferencias encontradas en las verbalizaciones sobre cómo cada profesor encara su oficio y enfrenta las prescripciones. Las situaciones comunes que estos profesores vivencian, resaltan las diversas interrelaciones con la diversidad de grupos de estudiantes en los turnos donde dan ellos clase, el cumplimiento de los procesos que implican enseñar, la evaluación de su desempeño y el control del gestor académico con ellos, por ser estos dos últimos elementos externos al trabajo que realizan y analizados subjetivamente por un tercero, bajo parámetros que no controlados y desconocidos por el profesor enseñante.

Estos encuentros, desvelaron los modos de operación de cada profesor, como elemento en que la actividad de trabajo es más expuesta, que a su vez, provocaron su reflexión al ser contrastados con el contexto universitario vigente y sobre el cual ningún profesor había pensado, considerado o reflexionado anteriormente, pues era considerado como natural, por lo cual se generó un elemento novedoso, para pensar sobre esta relación, tal como lo señaló P3, quien manifestó la naturalidad de tener que trabajar bajo esas condiciones y regímenes, lo que ello que implica para él y como pudiera ser mudado esa situación, como lo manifiesta: 
trabajo las tienen, esto es lo que hago desde hace un buen tiempo, pero al pensar sobre esto, ahora veo que este trabajo es visible sólo al dar dictar, pero esto sólo es una parte, yo trabajo mucho más en casa, estudiando, elaborando las clases, corrigiendo y poniendo notas, subiendo las clases al sistema, todo esto me causa que tenga que dejar de tener tiempo con mi familia, con mi hija pequeña, hasta de tener tiempo para descansar o estudiar una maestría que necesito hacer, pero es muy cansado, imagínese, yo enseño en varias universidades, en varios días, a veces hasta sábado, el domingo quiero hacer todo, el tiempo no alcanza, vivo en angustia, pero tengo que dar cuenta y doy cuenta, aunque con sacrificio". (verbalización P3).

Estas situaciones comunes encontradas al verbalizar los profesores sobre su trabajo, fueron los disparadores sobre los cuales, se empleó la ACS, procurándose conocer el doble movimiento del juego dialéctico de la actividad (SCHWARTZ, 2006), como posibilidades que el profesor encuentra para recrear y modificar las normas (aquellas relacionadas con la estructura de los contenidos, relaciones con los colectivos, el perfil docente, el reglamento interno, la ley universitaria, la concepción del docente universitario), así como las normas creadas por él para salir bien y responder a sus propios objetivos y a los objetivos institucionales (negociaciones con los estudiantes, con la coordinación académica, incorporar modificaciones en los sílabos, espacios para la formación política y ciudadana); además de conocer como su trabajo atraviesa otras dimensiones (personales, familiares, físicas, emocionales, etc.) y conocer el sentido y significado atribuido por él para seguir como profesor en la universidad.

Con estos elementos se generaron el diálogo y la interacción con el profesor, el cual no siempre fue espontáneo, pero necesario para el desvelamiento de aquello que no es visible y ausente de voz sobre su trabajo como enseñante, la configuración de su oficio, lo que hace, lo que deja de hacer, lo que percibe, lo que siente, sus dramas, sus angustias, las presiones que siente, el trabajo invadiendo otros espacios, su trabajo como aspecto económico y deontológico.

En esa construcción hermenéutica que las verbalizaciones y diálogos traen en ACS, esta no fue desarrollada apenas con un marco de preguntas que provocaron respuestas, sino como una invitación para que el profesor observe su trabajo, lo reviva, lo sienta y piense sobre aquello, sobre sus elecciones, trasgresiones y las nuevas creaciones elaboradas por él, conduciéndolo a considerar que su actividad no se estructura sólo por las normas, sino que en esas transgresiones se constituye la actividad como matriz de la historia humana en una dinámica que embiste a la estrecha articulación entre lo "imposible e invivible" de la reproducción idéntica de la actividad, constatando que la prescripción no ofrece los subsidios para hacer, sucediendo con ello permanentes renormalizaciones (EFROS, SCHWARTZ, 2009).

La ACS, aquí desarrollada no partió de un rutero de preguntas pre elaboradas, ella fue tomando su propia forma, en la medida, que la interlocución entre el investigador y el trabajador acontecía, en cuyo texto, se relataba una escena que compone una situación vivencial en el que por citar algunos momentos, como lo menciona P1 está presente: el cómo son enfrentadas las situaciones producidas entre aquellos estudiantes con experiencia laboral y aquellos que poseen está experiencia.

En el diálogo con P3, aparecen las acciones que él realiza para incorporar además de las sesiones de clase planeadas según silabo del curso (plano de clases), él incorporaba sesiones adicionales para ayudar a los estudiantes con dificultades para comprender los contenidos básicos, que dificultaban la transferencia pedagógica. 
Por su parte con P2, el diálogo se enfocó en como él, establece y negocia las reglas con aquellos estudiantes que no tenían la claridad de asumirse como universitarios y que manifestaban su desinterés por aprender los contendidos de las clases y participar con el desarrollo de las actividades colectivas.

En todas estas exploraciones la ACS posibilitó traer a escena vivencias, experiencias, saberes valores que el profesor le otorgaba voz y espacio para pensar sobre ello. Con ello, al revivir y pensar sobre sus acciones, las elecciones y arbitrajes realizados, el profesor vía la ACS, provocó rupturas sobre ese imaginario simbólico impuesto y que pretendía establecer sobre su trabajo una hegemónica concepción mercantil y privada de su actividad, recolocando el sentido y significado sobre su trabajo del profesor, colocándolo más cerca de comprenderla bajo la concepción de un oficio, de un bien público, donde el otro (estudiante) importa no apenas como cliente y por tanto su conocimiento no es apenas una mercancía; sino que con ello puede trasgredir su concepción de no sólo buscar un desempeño, sino una finalidad que más allá de valores económicos, que lo llevan a emanciparlo, al reflexionar sobre cómo, por qué, donde y para a quien lo ejerce.

Al ser la ACS un método nuevo para el profesor participante de la investigación, al ser conocida por primera vez por él, causó extrañamientos y resistencias para verbalizar al inicio sobre aquello que acontece en su trabajo. Mas, luego del acto de leer sobre sí mismo, sobre aquello que él hace y deja de hacer, generó en él, nuevos espacios para revivir las escenas que configuraban una experiencia situada, releer sobre aquello hecho y ahondar en algunos detalles que se escaparon del texto escrito, permitieron también constatar que en este movimiento, se deja expuesta y comprometida su intimidad. Así, al repensar sobre una determinada acción y revisitarla con más detalles provocó por ejemplo en el P3, que se quede sin palabras para explicar cómo, por qué lo hace y por qué deja de hacerlo.

Estas situaciones y experiencias con cada profesor, Roca (2017) señala que trae consigo una atención permanente, un manejo de disposición, tenacidad y paciencia para abordar situaciones en los que el diálogo no fluye o parece estancarse, pero que a su vez, son necesarios para comprender e ir en procura de reiniciar el diálogo y traer algunos entre telones de la actividad, el debate de normas y las renormalizaciones, puntos apuntados en líneas anteriores.

En suma, esta experiencia con el uso de la ACS bajo la perspectiva ergológica, colocó en evidencia las posibilidades aún por explorar de este método en lo referente a los usos de sí, las normas antecedentes, entre otros.

\section{CONCLUSIONES}

El trabajo forma la especie, el hombre y el individuo en términos de habilidades y competencias técnicas, políticamente, en la relación con procesos más amplios de socialización, relativos o no a los procesos de escolarización. Apenas una ínfima parte de esta experimentación funcional diaria da lugar a la experiencia cristalizada como aprendizajes en el trabajo. La experiencia de trabajo precisa ser elaborada en algún grado para emerger en cuanto aprendizaje, ¿pero sería el trabajo educativo en 
estos varios sentidos solamente cuando son pasados en revista por nuestra capacidad analítica?, es una cuestión filosófica y epistemológica en abierto.

La autoconfrontación en sus varias vertientes abre este proceso de análisis del trabajo en la medida en que invita al extrañamiento entre lo es realizado realmente y todo que atraviesa y estructura el ejercicio de trabajar. No es toda la globalidad de esta experiencia (cuyos límites no son debidos a la incompetencia lingüística de aquellos que trabajan), es constructivista en la medida en que podemos hablar en desarrollo y fortalecimiento de la experiencia profesional, puesto que trae a la conciencia labora por qué y el cómo funciona el sistema socio técnico en el cual la actividad está inmersa, bien como desvela algunas curvas de la propia actividad para el sujeto que trabaja. Un proceso de desnaturalización de la actividad es, por tanto, desencadenado por la autoconciencia del sujeto, más allá de lo que fue programado, hay siempre la intervención de quien trabaja.

Esta conciencia es el desplazamiento en la precepción de aquello que es el trabajo, sus normas productivas, técnicas económicas, etc., y como, en alguna medida el trabajador se coloca frente de ellos consciente o inconscientemente. La interactividad entre trabajadores sobre el propio trabajo y de estos investigadores con el investigador puede representar una desestabilización en relaciones sociales cristalizadas en situaciones de trabajo analizadas al desvelar las dimensiones subterráneas de esta experiencia, la cual, tomada sin este proceso de autoconfrontación, la experiencia puede venir poco problematizada perdiendo el interés del punto de vista de la transformación del trabajo. Mas la experiencia así problematizada, por el hecho de hacer emerger los aprendizajes formales o informales construidos a la prueba de las situaciones de trabajo, entre tanto, está más apta a ser procesada por quien la sostiene en otros contextos. Es por eso que este proceso es recordado por sus cualidades formadoras para el trabajador, sea porque valoriza su punto de vista en las transformaciones y mejorías en el trabajo (ODONNE, 2001), sea porque lo instruye por el discernimiento sobre los debates de normas que atraviesan todo trabajo (SCHWARTZ, 2006), sea porque desvela las dimensiones subjetivas que saturan los gestos profesionales (CLOT \& FAÏTA, 2000).

La formalización de esta experiencia diaria exigirá, por tanto, esfuerzo de aquel que busca hacer, no apenas un dominio técnico de aquellos que buscan implementarlo en cuanto recurso metodológico. Y puede, como en el caso de la clínica de la actividad, ser considerada un método, pero no el único, para acceder a la parte íntima de la experiencia de trabajo que se actualiza e integra en los gestos laborales.

Del punto de vista de la producción del conocimiento, ese material, rico en experiencia laboral, puede ser problematizado en sentido de la construcción de otra producción científica sobre el trabajo. En esta perspectiva, lo que podemos comprender del trabajo realizado no puede ser sin la contribución de estos, aún que este autoanálisis del trabajo no pueda ser agotada, puesto que no es toda la experiencia que es analizable, bien como este proceso no pueda prescindir del diálogo, no menos, con las contribuciones de los diversos campos del conocimiento que estudian el trabajo.

Es lo que podemos aprender de estos linajes de investigaciones clínicas sobre el trabajo que, más allá de experimentación metodológica en el sentido de adaptación del hombre al trabajo, abrió un campo de reflexión ético-política y epistemológica en el cual cualquier mejoría en las situaciones de 
trabajo se debe colocar en el horizonte de promoción de salud y del bien estar de aquellos que trabajan.

\section{REFERÊNCIAS}

1. ALVES, V.A. A atividade de trabalho docente em uma escola privada: Usos de si e circulações de valores, saberes e competências. (Dissertação de mestrado em educação). Programa de PósGraduação em Educação: Conhecimento e Inclusão Social. Universidade Federal de Minas Gerais. 2009.

2. BRONCKART. Louvain-la-Neuve : BCILL, 2005, p. 5-16.

3. CHOMSKY, N. O neoliberalismo tomou de assalto as universidades, 2014. In: El démarche ergológico en el análisis de la actividad del profesor universitario en sala de aula. Revista Docência do Ensino Superior, v. 8, n. 1, p. 27-42, 11 jul. 2018.

4. CLOT, Y. \& FAÏTA D. Genre et style en analyse du travail. Concepts et méthodes. Travailler, $\mathrm{n}^{\circ} 4$, 7-42. 2000

5. CLOT, Y. et al. Entrétiens en autoconfrontation croisée : une méthode en clinique de l'activité. In : Éducation Permanente, n. 146, 2001, p. 17- 25.

6. CLOT, Y. et al. L'autoconfrontation croisée en analyse du travail : l'apport de la théorie bakhtinienne du dialogue". In : L'analyse des actions et des discours en situation de travail : concepts, méthodes et applications. Sob a direção de L. FILLIETTAZ e J.-P. CLOT, Y. Trabalho e poder de agir. Belo Horizonte: Fabrefactum, 2010.

7. DOUNIS, A. B., Et al. A autoconfrontação: um estado da arte das produções acadêmicas disponibilizadas na biblioteca digital brasileira de teses e dissertações da CAPES. In. XVI ENDIPE Encontro Nacional de Didática e Práticas de Ensino - UNICAMP - Campinas - 2012.

8. EFROS, D.; SCHWARTZ, Y. Résistances, transgressions et transformations:'impossible invivable dans les situations de travail. In: Nouvelle revue de psychosociologie, v.1.n.7, p. 33 - 48, 2009. Disponible en: < https://www.cairn.info/revue-nouvelle-revue-de-psychosociologie-2009-1-page33.htm >. Consultado el 12 mayo de 2018.

9. FERNANDEZ, G. CLOT. Y. Entrevistas en auto-confrontación: un método en clínica de la actividad. Informática na educação: teoria \& prática. v.13, n.1, jan./jun. Porto Alegre, 2010. 
FERNANDEZ, G. CLOT. Y. Entrevistas en auto-confrontación: un método en clínica de la actividad. Informática na educação: teoria \& prática. v.13, n.1, jan./jun. Porto Alegre, 2010.

11. LOUSADA, E. G. Entre trabalho prescrito e realizado: um espaço para a emergência do trabalho real do professor. Tese (doutorado em educação). Pontifícia Universidade Católica de São Paulo, 333 f., 2006.

12. MACHADO. V. Et al. Contribuições da autoconfrontação para o estudo da atividade docente. In VI Colóquio. Educação e contemporaneidade. 2012.

13. MUNIZ, M.I. A. Autoconfrontação simples: condições de produção e autoconhecimento. Alfa, 54 (1): 81-111, São Paulo. 2010.

14. ODDONE, I.; RE, A.; BRIANTE, G. Redécouvrir l'expérience ouvrière: vers une autre psychologie du travail? Paris: Éditions Sociales, 1981

15. ROCA, D.F.R.F. El trabajo del profesor en una universidad peruana originaria de un movimiento estudiantil. Tesis presentada para obtener o Grado de Doctor en Educación. Faculdade de Educação. Universidade Federal de Minas Gerais. Belo Horizonte. 336 f. 2017.

SCHWARTZ, Y. A linguagem no trabalho. [Trad. Cecília Souza-e-Silva e Décio Rocha]. In: Schwartz, Y. \& Durrive, L. (org). Travail et ergologie: entretiens sur l'activité humains. Toulouse: Octarès, 2006.

17. SCHWARTZ, Y.; DURRIVE, L. (Org.). Trabalho \& Ergologia: conversas sobre a atividade humana. 2. ed. Niterói: Editorial UFF, 2010. 309 p.

18. SCHWARTZ, Y.; DURRIVE, L. (Org.). Trabalho \& Ergologia II: diálogos sobre a atividade humana. Seguido de manifesto por um ergoengajamento. Ed. Fabrefactum, 2015. 396 p.

\section{Daisy Moreira Cunha Correio}

Professora Doutora do Departamento de Educação da UFMG.

\section{Daniel Fabián Roca Flores Pinto}

Doctor en Educación por la FAE/UFMG. Magíster en Docencia en la Educación Superior e Investigación por la UPCH. Graduado en Administración por la UIGV. 


\section{Maria leda Almeida Muniz}

Professora Doutora pelo LAEL/PUC-SP. Trabalha como professora associada da UTFPR no Departamento de Letras.

\section{Como citar este documento:}

CUNHA, Daisy Moreira; PINTO, Daniel Fabián Roca Flores; MUNIZ, Maria leda Almeida. El trabajo y el desenvolvimiento laboral por autoconfrontación. Reflexão e Ação, Santa Cruz do Sul, v. 28, n. 3, p. 2234 , ago 2020. ISSN 1982-9949.

Disponível em: <https://online.unisc.br/seer/index.php/reflex/article/view/13672>. Acesso em: doi:https://doi.org/10.17058/rea.v28i3.13672. 